\title{
DYSMORPHIC PATIENTS: A REVIEW OF MINOR CLINICAL SIGNS LEADING TO A DIAGNOSIS
}

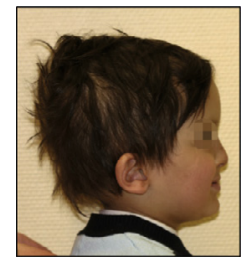

\section{Muriel DE LA DURE-MOLLA, Pascal GARREC}

\begin{abstract}
The dental surgeon can feel inadequate when confronted with a rare disease: (a) difficult diagnosis, (b) therapeutic complexity, (c) and questioning by the parents, are three major obstacles that we have to overcome in order to carry out our therapy. Even if the field of genetics has made major advances during the last few years, it is clinical knowledge, above all, that leads us to a diagnosis. Certain syndromes present with very subtle signs which alone can appear trivial but which, taken together, defines a pathological entity. To know or to discover these signs and to recognize them is therefore the first challenge.
\end{abstract}

KEY WORDS

Oligodontia

Syndrome
Dysmorphia

Dental anomalies

\section{INTRODUCTION}

The family questionnaire is a major initial part of the appraisal of the patient, leading to the suspicion of a genetic disease. The genealogical tree leads to an understanding of the patient's mode of inheritance and therefore helps to determine how to proceed during the genetic investigation. For example, when several children from the same family are similarly affected (called horizontal transmission), a recessive transmission can be inferred to explain the absence of the genetic phenotype in the parents. Likewise, when several boys are affected on the maternal side, this would suggest that transmission is linked to the $X$ chromosome. A dominant 
transmission is often recognized when we encounter a vertical transmission with incomplete penetrance. Finally, if the patient is the first to present the mutation, we refer to this as de novo and as a sporadic case. Advanced paternal age is one element that favors this type of de novo mutation.

It is also important to evaluate the history of pregnancies. The his- tory of previous miscarriages can be the result of a familial chromosomal abnormality. A thorough pediatric clinical history of the child can reveal the appearance of common pathologies (constipation, eczema, fevers...) but which, taken together, are all indications of the same malformative clinical entity.

\section{1 - THE CLINICAL EXAMINATION OF THE CHILD: SPOTTING THE SIGNS OF THE DYSMORPHIA}

The examination of the face is rich with information in many cases of malformative pathologies. The examination may however be subjective in the absence of a methodical and benchmark-based analysis ${ }^{7}$.

The objective of the clinical examination is to research the major criteria (malformations) and minor criteria (more minor morphological variations). Different anatomical elements need to be examined. Photographs are an important element of this exam and often allow us to follow the dysmorphia throughout growth. The examination of the cranio-facial complex includes a series of steps ${ }^{1}$.

\section{Examination of the cranium}

Full face, profile and superior views should be taken. The practitioner should look for symmetry and general proportions. Anomalies of the shape of the cranium (dolichocephalic, plagiocephalic...) are generally minor anomalies. Certain pathologies however like craniostenoses (an anomaly of the closure of the cranial sutures) are associated with significant cranial dysmorphias that become one element of the diagnosis.

\section{Examination of the face}

This allows the measurement of the facial proportions: The face is divided into 3 thirds, defined by landmarks that vary from childhood to adulthood but which normally retain equivalent height proportions. The mobility of the facial muscles needs to be evaluated as well. An elongated and unanimated face is characteristic of facial myopathy, for example.

\section{Upper third}

One starts by evaluating the forehead (high/low, wide/narrow, convex/ flat, vertical/receding, smooth/wrinkled). One starts by examinating, then, the peri-orbital region (examine the palpebral fissures, the orbits, the eyeballs and eyelashes) (Fig. 1). 


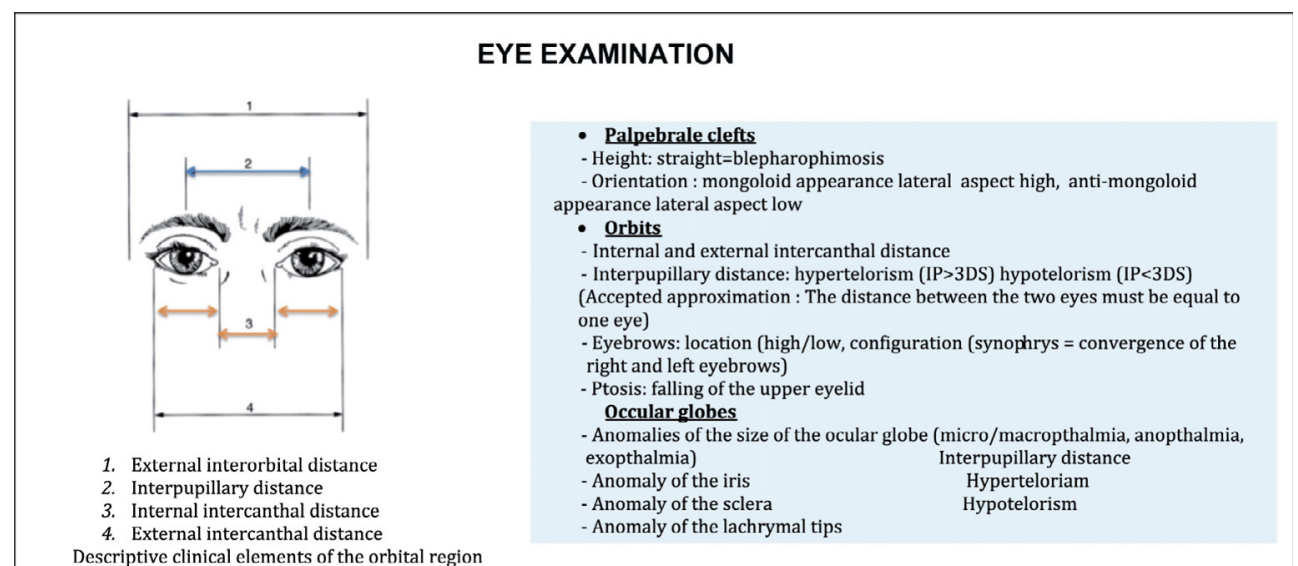

- Orientation

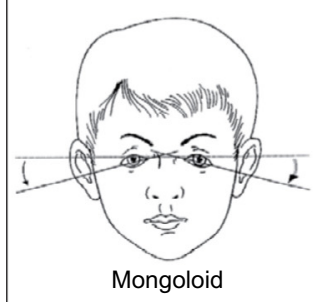

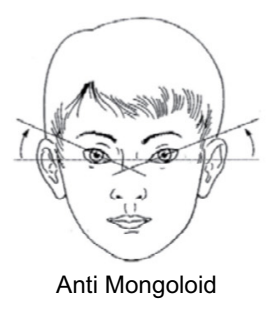

- Interpupillary distance

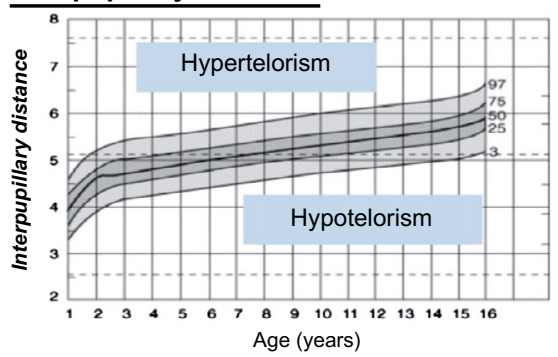

Figure 1

Clinical examination of the eyes.

\section{Middle third}

This examination is made in both full face and in profile views. When examining the nose, the practitioner should look at the nasal saddle, the root (slope) of the nose (flattened, depressed, protruding, wide, narrow), the alae (wings) of the nose (thick, hypoplastic), and the nostrils. Finally one should examine the philtrum that can be shorten in numerous syndromes (Fig. 2). The ears are also a part of the middle third of the face. Note their sizes, (large/small), their placement (high/low), their orientation (rotation posterior or anterior) and shape (helical, antihelical, tragus, antitragus, lobule, concha...) (Fig. 3).

\section{Lower third}

The examination is made from the lips to the chin. Thin lips for example are, among others, characteristic of fetal-alcohol syndrome. A study of the chin provides information on the shape and position of the mandible. Antero-posterior and vertical anomalies can be assessed by in studying the alignment of the foreheadphiltrum and chin. An examination of the oral cavity will finish the study of this region (Fig. 4).

\section{Examination of the hair}

Examination of the hair is part of the evaluation of the appendages 


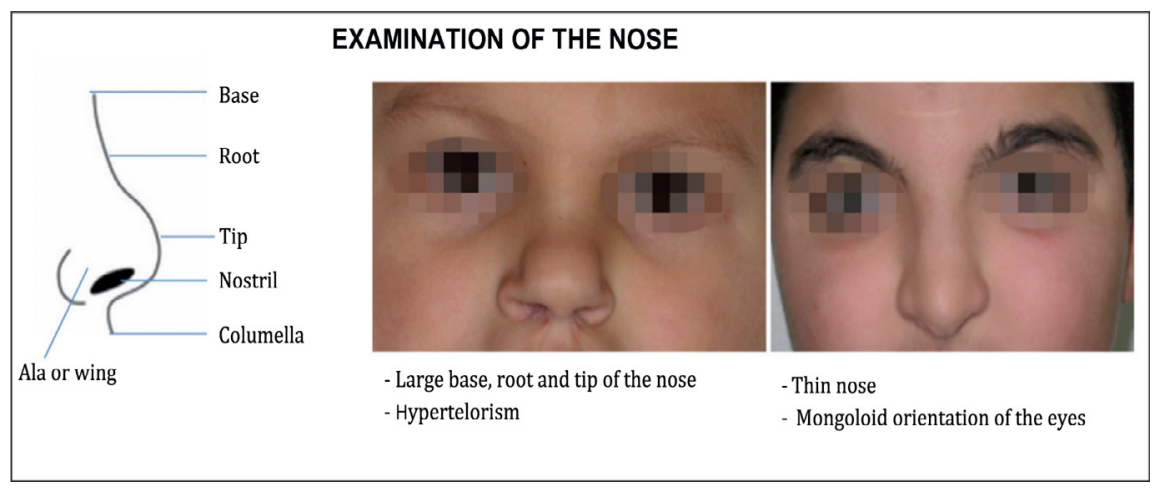

Figure 2

Clinical examination of the nose.

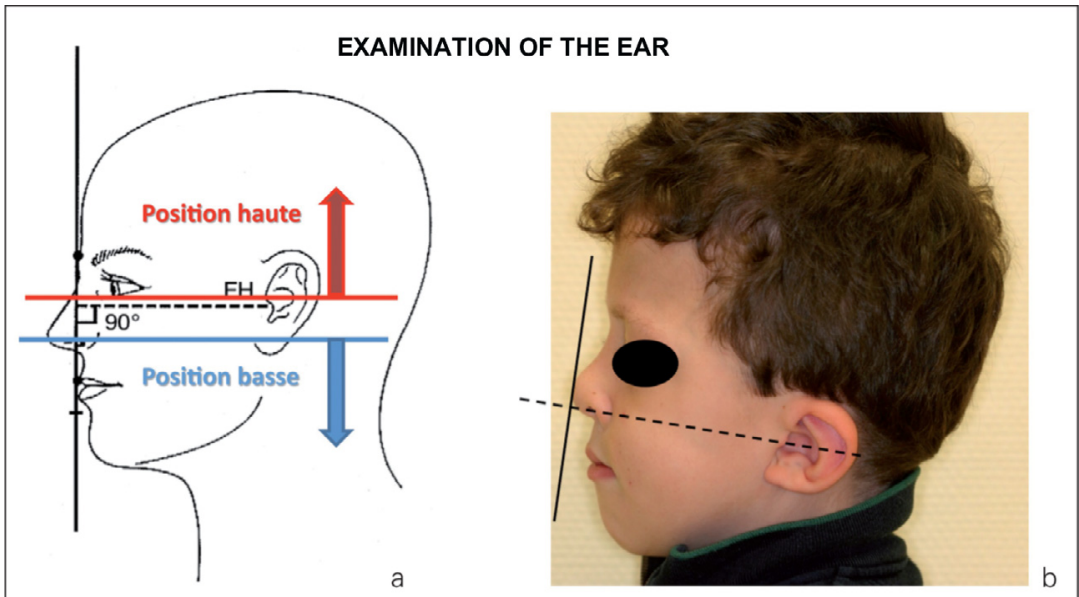

Figure 3

Clinical examination of the ears:

a: normal position of the ear; $b$ : patient presenting a lower position and a posterior rotation of the ear.

The position of the ear is determined by the intersection of two lines:

- A vertical line joining the glabella and the most anterior superior labial point

- A horizontal line perpendicular to the previous passing by the Porion point (tragus)

The position of the ear is normal if these two lines cross each other in the zone located

between the part that is superior to the wing of the nose and the part that is inferior to the lower eyelid.

(structures intimately linked to the teeth from an embryological perspective). We note the quantity (hypotrichosis, hypertrichosis, alopecia), the quality (fine hair, brittle...) and the presence of hair on the forehead (brow) or the neck.

\section{Examination of the hands}

This examination consists of an analysis of the shape and an analysis of the cutaneous (skin) covering (Dermatoglyphs). Dermatoglyphic analysis (finger patterns and ridge counts) consists of an analysis of the palmoplantar skin folds as well as the fingers, and fingertips. The presence of a single transverse palmar crease can be a symptom associated with abnormal medical conditions, such as fetal alcohol syndrome, or with genetic chromosomal abnormalities, including Down Syndrome (trisomy 21). 


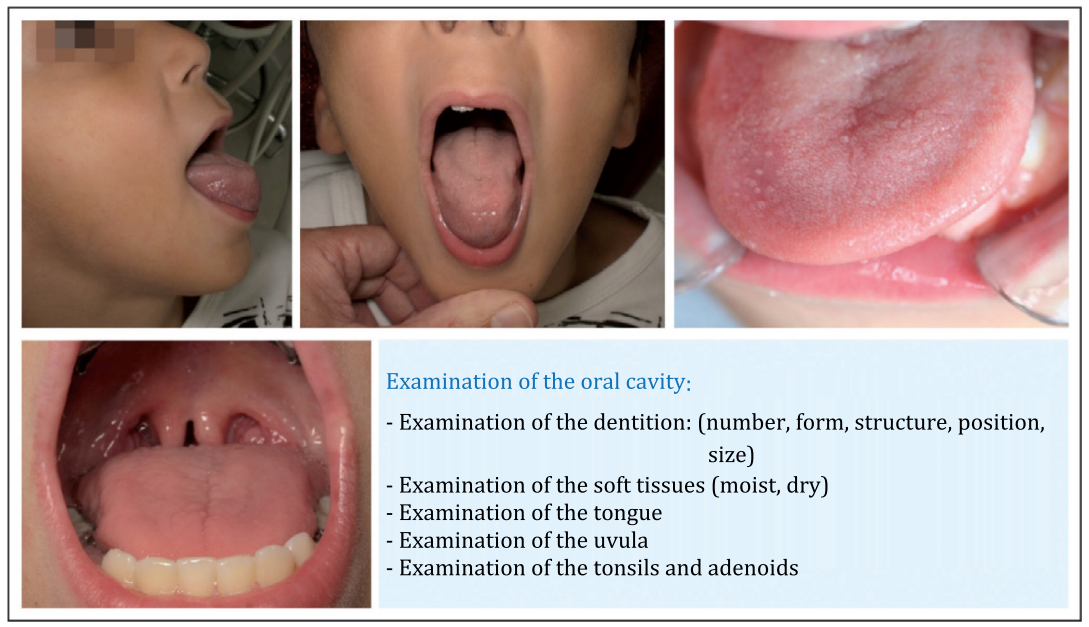

Figure 4

Clinical examination of the oral cavity.

During the inspection of the hands (and feet), the practitioner initially assesses the dimensions, checks for signs of syndactyly and analyzes flexibility and length of phalanx (distal, medial, proximal) (Fig. 5) ${ }^{4}$.

\section{Psychomotor and intellectual development}

Many dysmorphic syndromes are accompanied by psychomotor and/ or behavioral problems to varying degrees. The behavioral phenotype

\section{2 - DENTAL AGENESIS}

Very few other causes than genetics interfere with the early development of the dental buds except for chemotherapy during the first years of life. More than fifty mutated genes have already been described in humans. Only 2 or 3 are responsible for an isolated agenesis. In these cases, the patient presents with a single and unique phenotype for the dental agenesis. In all other cases the gene has a greater impact on embryological requires a systematic examination. It can be specific in certain syndromes and is therefore a diagnostic criterion. The examination seeks to evaluate:

- its progressive nature (regressing or stable);

- language features;

- attention deficits;

- specific behavior (stereotypical...);

- social behavior;

- sleeping problems.

development and dental agenesis is but one sign among others that are more or less severe ${ }^{5}$. Ectodermal dysplasia is a more frequent example.

\section{Ectodermal dysplasias (Fig. 7)}

\section{Oro-facial signs:}

- dental agenesis of the primary and permanent teeth;

- dry mouth; 


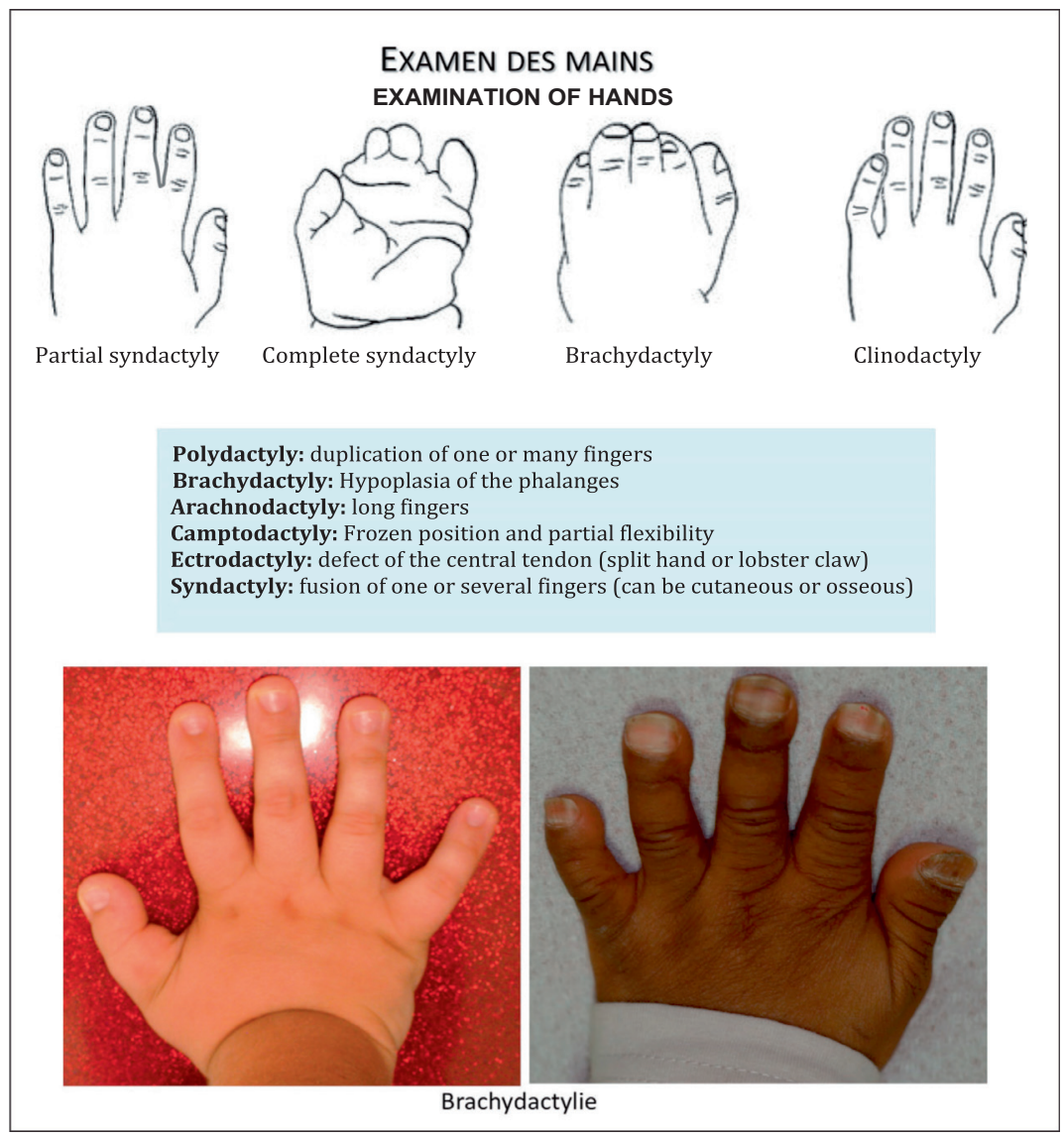

Figure 5

Clinical examination of the hands.
- microdontia;

- conical shaped anterior teeth;

- hypotrophic alveolar ridges;

- Reduced height of the lower facial third.

\section{Clinical extra-oral signs:}

- cutaneous: dry skin (ichthyosis), atypical dermatitis (eczema, allergies),

- hyperkeratosis (thickening of the skin) of the palms of the hands and the soles of feet,

- fine hair, sparse, brittle and dry (hypotrichosis),

- thin eyelashes,
- thin eyebrows (mostly on the external third),

- respiratory (rhinitis with dry mucosa, asthma),

- sensation of dry eyes, photophobia (reduction of lachrymal fluid).

- constipation.

Ectodermal dysplasias are grouped into more than 180 pathological entities affecting one or more ectodermal derivatives: nails, hair, sweat glands and teeth. The effect of a single derivative corresponds to a group of pure dysplasias. The effect on another organ (eye, ear, palate, nervous system...) defines syndromic ectodermal dysplasias. The most frequent form is anhydrous ectodermal 


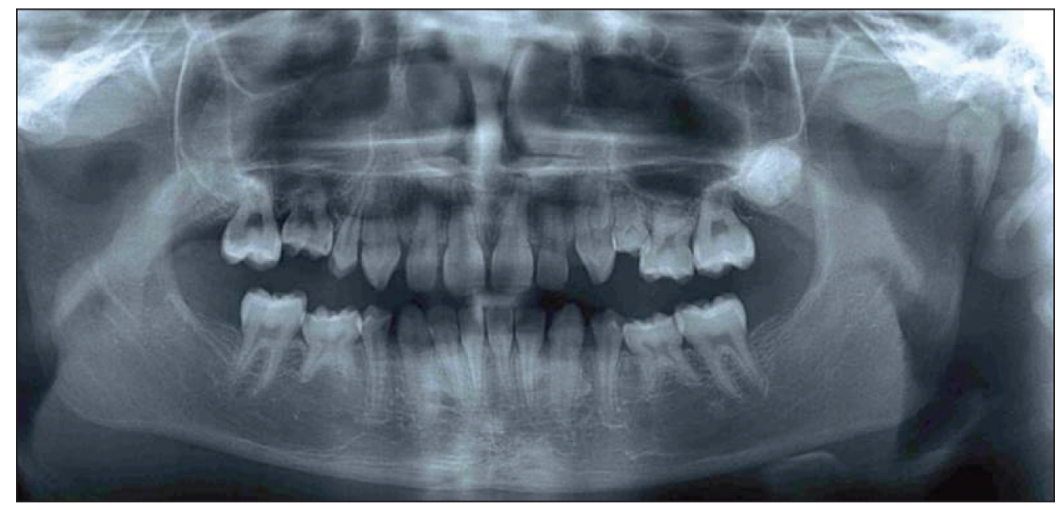

Figure 6

Panoramic radiograph of a 12 year old child having received chemotherapy treatment for a neuroblastoma at 6 years of age having dental consequences: agenesis of permanent teeth and short roots (in the permanent incisor area).

dysplasia. Reduction through absence of sweating is the main characteristic. In the most severe forms, if the diagnosis is not made at birth, there is the risk of death from hyperthermia. The lack of sweating is the most handicapping symptom in the daily lives of these patients. There presently is no treatment, but counseling is indispensible: live in an environment where the temperature does not exceed $23-24^{\circ} \mathrm{C}$, hydrate, avoid sports or any activities that are prone to increase body temperature. The effect on the hair is constant and impacts all the zones. The lack of secretion from all mucosal areas is responsible for different effects (respiratory, ocular, digestive). Regardless of the sign, the severity of the effect can be variable. At the dental level it is just the same. Some patients present only with hypodontia (fewer than six missing teeth) while other have anodontia in both jaws. The anterior teeth have a pathognomonic conical shape. This variability comes from the nature of the mutation and mostly from the affected gene. There exists today more than 30 causative genes $^{12}$.

\section{How to proceed}

When the primary teeth are missing in the arch, a panoramic $x$-ray should be taken starting at 5 years of age to evaluate the permanent teeth that are present. Prior to this age the $x$-ray is not instructive because the buds for the premolars, and second molars are minimally or not yet mineralized.

\section{Odonto-onycho-dermal dysplasia} (OODD Syndrome) (Fig. 8)

\section{Clinical oro-facial signs:}

- Severe oligodontia;

- Conical anterior teeth;

- Reduction in the lingual papillae (filiform and fungiform).

\section{Extra-oral signs:}

- malformation of the nails (thin, brittle, convex, duplicated, slow growing) 

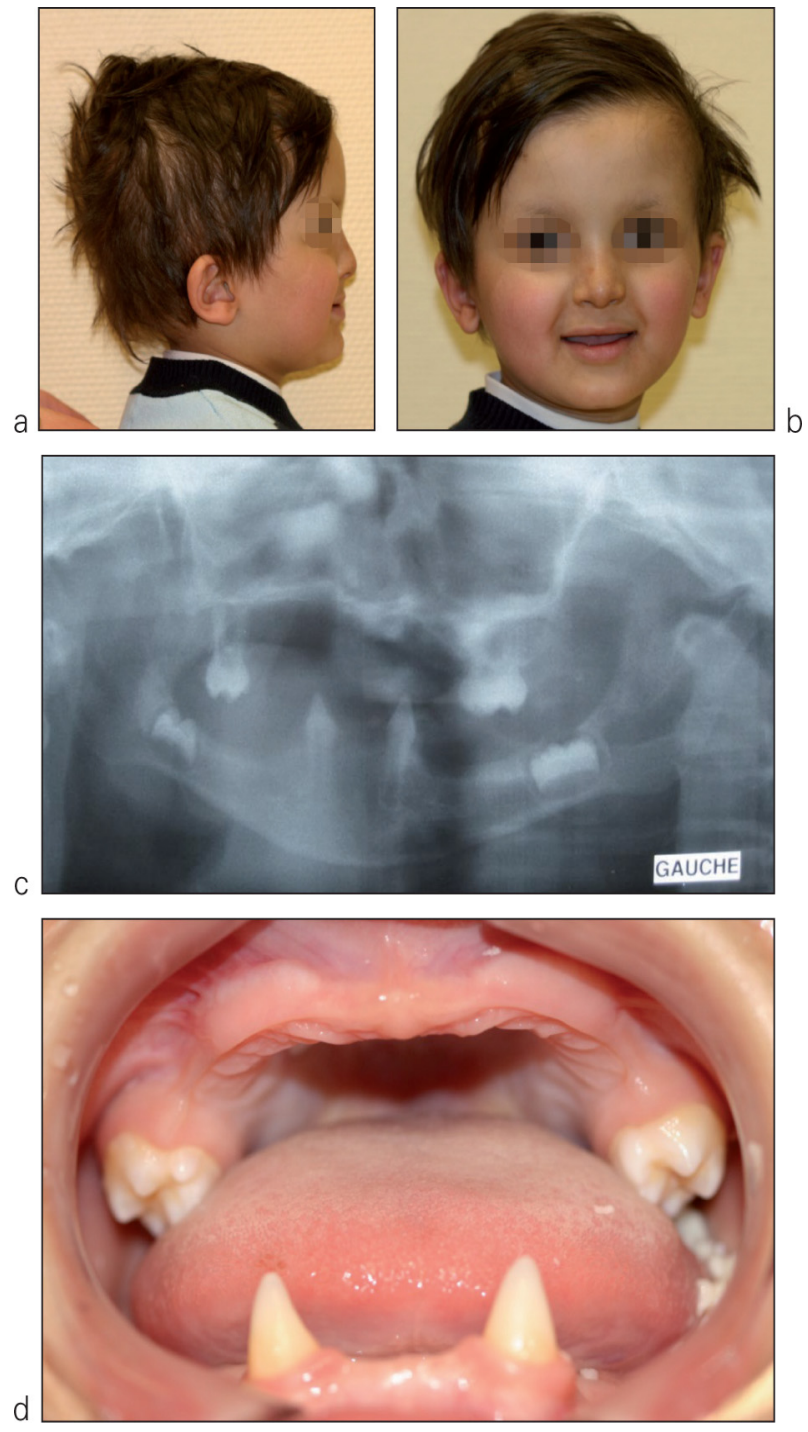

Figure 7

Patient presenting with hypohidrotic ectodermal dysplasia:

a \& b: photograph of the face and profile showing hypotrichosis and thin eyebrows;

$c$ : panoramic radiograph highlighting oligodontia in the primary and permanent dentition;

$d$ : Intra-oral photograph showing the cone shaped canines of the primary canines.

- mild skin dryness,

- atopic skin,

- frequent allergies,

- hypotrichosis,
- hyperkeratosis of the palms and soles of the feet,

- Hyper-sweating.

This syndrome has for a long time been considered as a mild form of ectodermal dysplasia, because of two effects derived from the ectoderm, On the other hand we didn't find any reduction in sweating but, on the contrary, excessive sweating. Recently, mutations of the gene WNT10A have been described and have thus made it possible to determine that this syndrome is a distinct pathological entity. This syndrome is transmitted whether with an autosomal dominant or recessive pattern. The recessive forms are generally more severe than the dominant forms. If the parents are not consanguineous, it is possible to find composite mutated heterozygotes where each parent is a carrier (without a single sign even a weak one). The clinical signs are very variable ranging from a quasi-isolated oligodontia through forms that express the entire group of extra-oral clinical signs.

The most severe clinical form are found in patients who present with a mutation of this gene. They constitute the Schopf-Schulz-Parsage Syndrome. They have the entirety of dental and extra-oral signs as well as cysts of the eyelids ${ }^{3}$.

\section{EED Syndrome (ectrodactyly ectodermal cleft syndrome) (Fig. 9)}

This syndrome associates an ectodermal dysplasia, a palatal cleft and an ectrodactyly. The causative gene (TP63) codes for a factor of transcription implicated in the development of ectodermal derivatives ${ }^{10}$. 

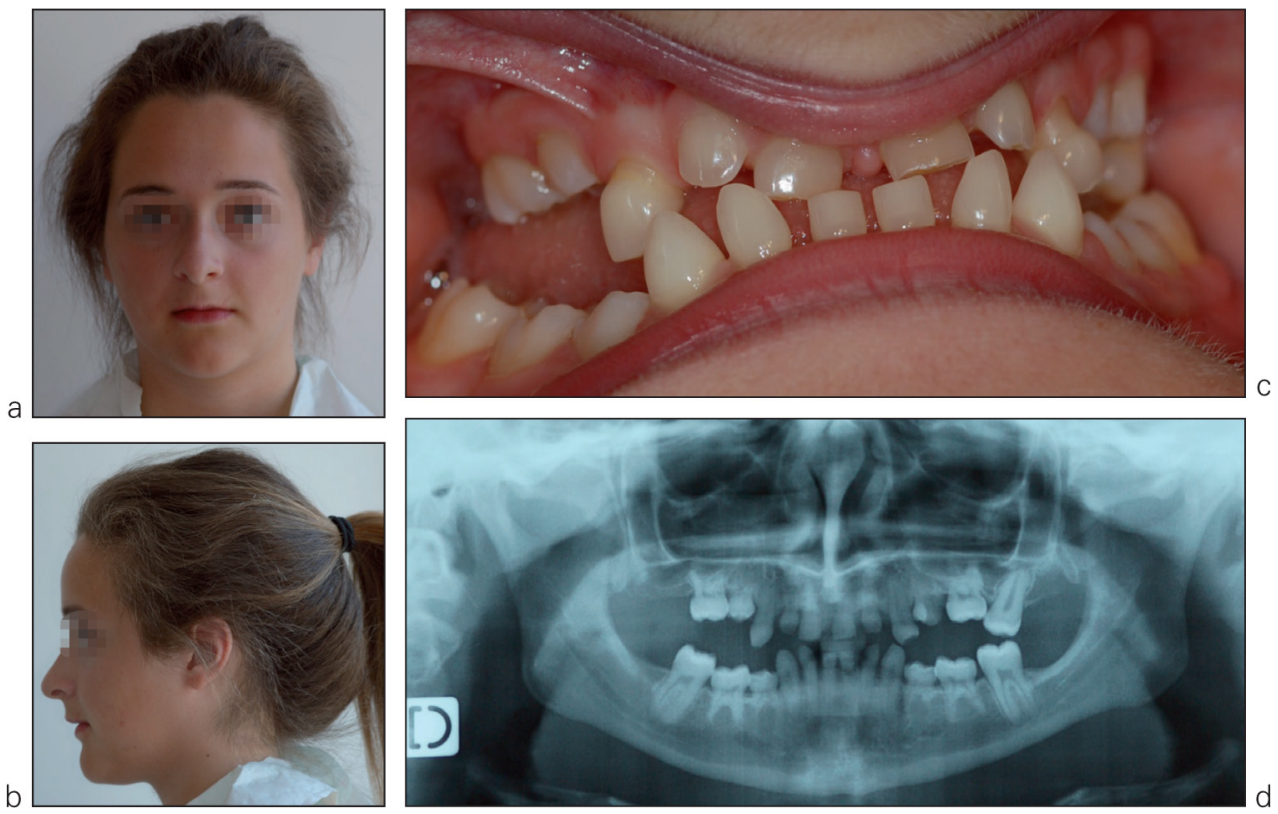

Figure 8

17 year old patient presenting with OODD syndrome:

a \& b: photograph of the face and profile

$c \& d$ : intraoral photograph and panoramic radiograph highlighting severe oligodontia of permanent teeth (29 missing teeth).

No familial history was found.

\section{Oro-facial clinical signs:}

- labio-palatal or palatal cleft;

- dental agenesis;

- ocular anomalies;

\section{Extra-oral clinical signs:}

- anomalies of the hands (ectrodactyly, syndactyly);

- ectodermal dysplasia;

- urogenital anomalies;

- deafness;

- hypoplastic mammary glands;

- choanal atresia (lack of posterior nasal openings);

- endocrine anomalies.

\section{Other syndromic forms}

Although it is the most frequent, ectodermal dysplasia is not the only syndrome associated with dental agenesis. To name a few:

- Axenfeld-Rieger syndrome is characterized by a malformation of the anterior chamber of the ocular globe. The clinical signs are generally: ocular anomalies (hypoplasia or deformation of the iris, corneal and anterior chamber anomalies, increased intra-ocular pressure, glaucoma...), anomalies of the umbilicus, and a discrete cranial-facial dysmorphia 

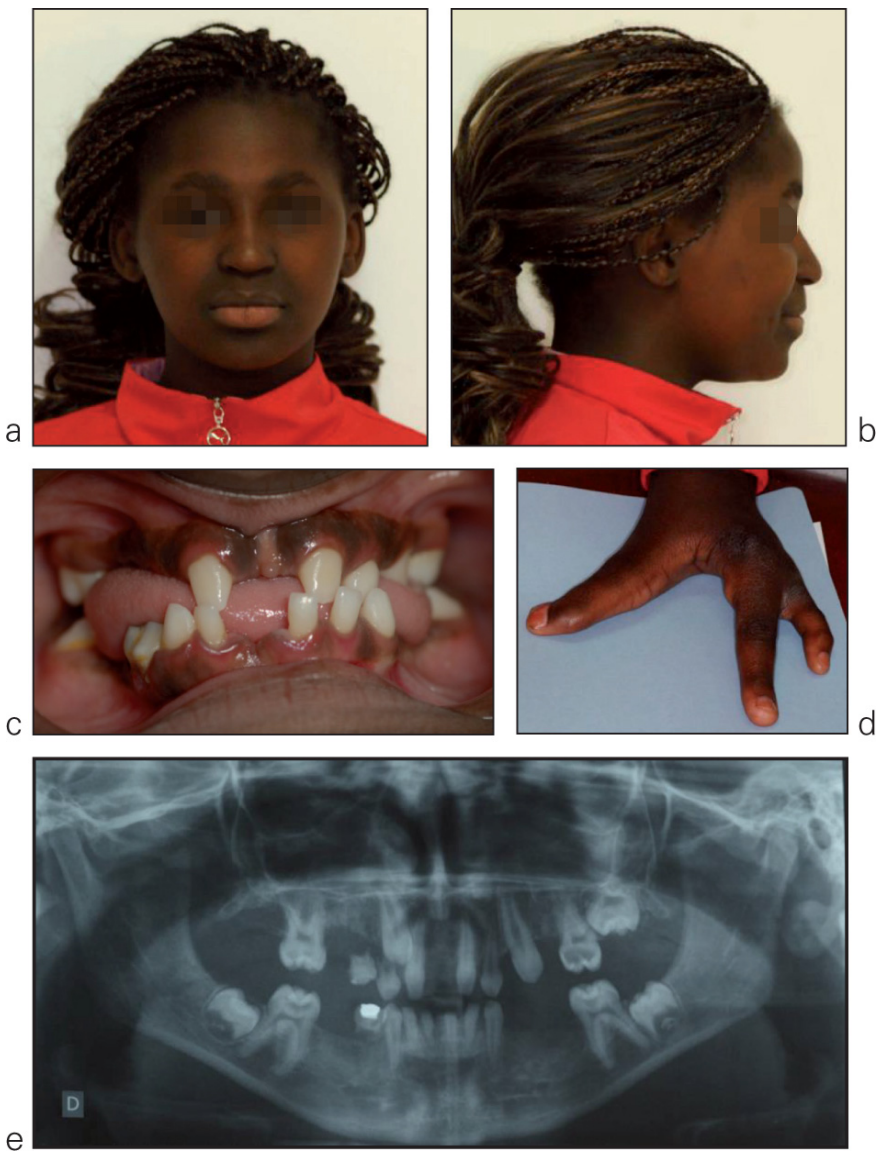

Figure 9

11 year old patient presenting with EEC syndrome:

a \& b: photograph of the face and profile showing an absence of eyebrows in the outer third;

c: intraoral photograph showing a microdontia of the maxillary central incisors, anomalies of position secondary to dental agenesis;

$d$ : ectrodactyly of both hands;

e: panoramic radiograph highlighting oligodontia of the permanent dentition.

(prominent frontal area, hypertelorism, flattened midface, hypodontia and microdontia).

- One central incisor syndrome is characterized by the agenesis of one central incisor of the primary teeth and the permanent without knowing whether it is the right or the left. The remaining incisor is found perfectly in the midline. The medial labial frenum is also missing. No other anomaly is found but it is advisable to refer this patient to a neurologist in order to investigate all the midline organs.

- OFCD syndrome (oculo-facialcardi-dental) is characterized by 
multiple congenital anomalies of the eyes (congenital cataracts, microphthalmia, eyelid ptosis, exotrophy), cardiac defects (inter-auricular communication, minor cardiomegaly, ventricular or auricular hypertrophy, benign peripheral pulmonary stenosis

\section{3 - ENAMEL ANOMALIES}

Among the anomalies of dental tissues enamel is the most frequently affected one. Many acquired etiologies are possible (excessive fluoride intake, Vitamin D deficiency, traumatism of the tooth bud...) but some genetic etiologies also exist (Fig. 11).

\section{Oro-facial clinical signs:}

- generalized hypoplasia of the enamel of all the teeth of both jaws;

- yellow/brown colored enamel;

- sensitivity of the teeth on mastication;

- pulpal calcification;

- delayed eruption;

- intra-coronal resorption;

- hyperplasia of dental follicles (hamartomas).

\section{Extra-oral clinical signs:}

- nephrocalcinosis

The patient presents kidney- tooth syndrome ${ }^{6}$. This syndrome associates an amelogenesis imperfecta with a nephrocalcinosis. Nephrocalcinosis consists of small volume ectopic calcifications in the kidney. It is and a mitral valve prolapse) and dental anomalies (late dental eruption, shape anomalies, long roots, taurodontism, agenesis). Large dental roots is pathognomonic of the syndrome (Fig. 10).

\section{- Predisposition to colorectal cancers.}

asymptomatic in the child and doesn't alter renal function. On the other hand, with ageing, it can transform into renal insufficiency necessitating other treatment plans. Regular follow-ups and a a referral to a pediatric nephrologist is mandatory. The mutated gene for this pathology, FAM20A, codes for a protein whose function is not yet well understood. On the other hand, the mice who do not express this gene present with multiple ectopic calcifications that are vascular, cerebral...

Hereditary Amelogenesis imperfecta (HAl) is a genetic disease that alters the development of the enamel. This pathology is characterized by qualitative changes (hypo mineralization, hypo maturation) or quantitative (hypoplasia) of the enamel of all the teeth. The teeth when they are erupting are already affected and this is what allows the establishment of the differential diagnosis along with all the other afflictions having a systemic origin. There exists a great variability of clinical expression in $\mathrm{HAl}$. The enamel is discolored going from chalky white to yellow brown. The loss of enamel tissue can be localized or generalized. Its hardness 

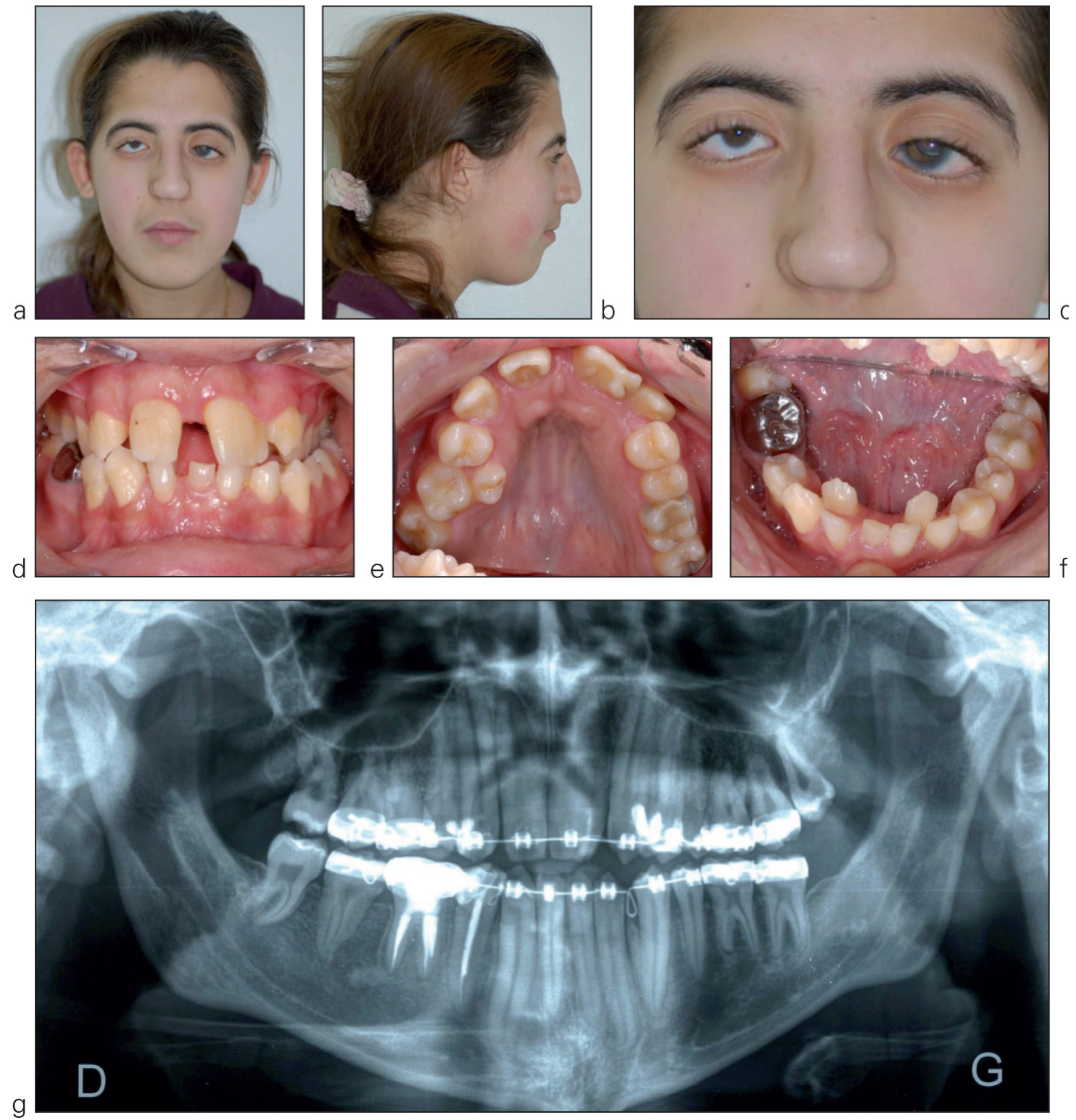

Figure 10

Patient presenting with OFCD syndrome:

$a, b$ \& $c$ : photograph of the face and profile showing a long face, a long nasal root, thin eyebrows with a lateral curve, a long philtrum, an exotropia and an exopthalmia;

d,e \& f: intraoral photograph showing anomalies of the shapes of 11 and 21 (macrodontia and talon cusp), malpositions and a lateral cross-bite;

g: panoramic radiograph showing a hypodontia and a radiculomegaly of the canines. 

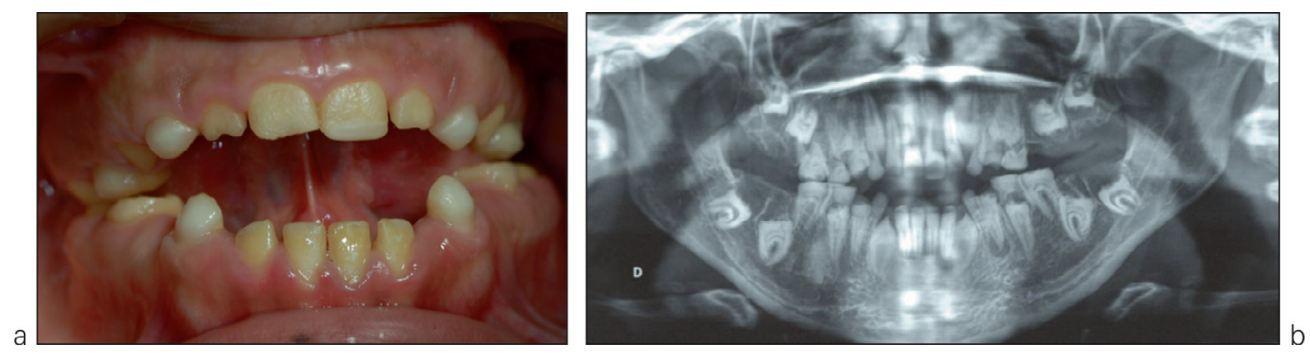

Figure 11

21 year old patient presenting with kidney-tooth syndrome:

a: intraoral photograph showing severe generalized enamel hypoplasia, associated with a pseudo-microdontia, a hyperfribromatosis and significant wear on the primary molars;

b: panoramic radiograph showing numerous unerupted permanent teeth, some generalized intra-pulpal calcifications, some permanent molar root shape anomalies as well as intracoronal resorption of impacted maxillary molars.

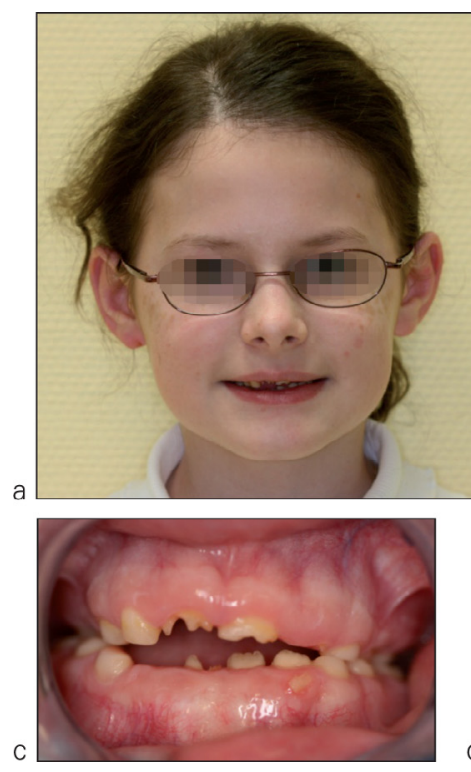

.
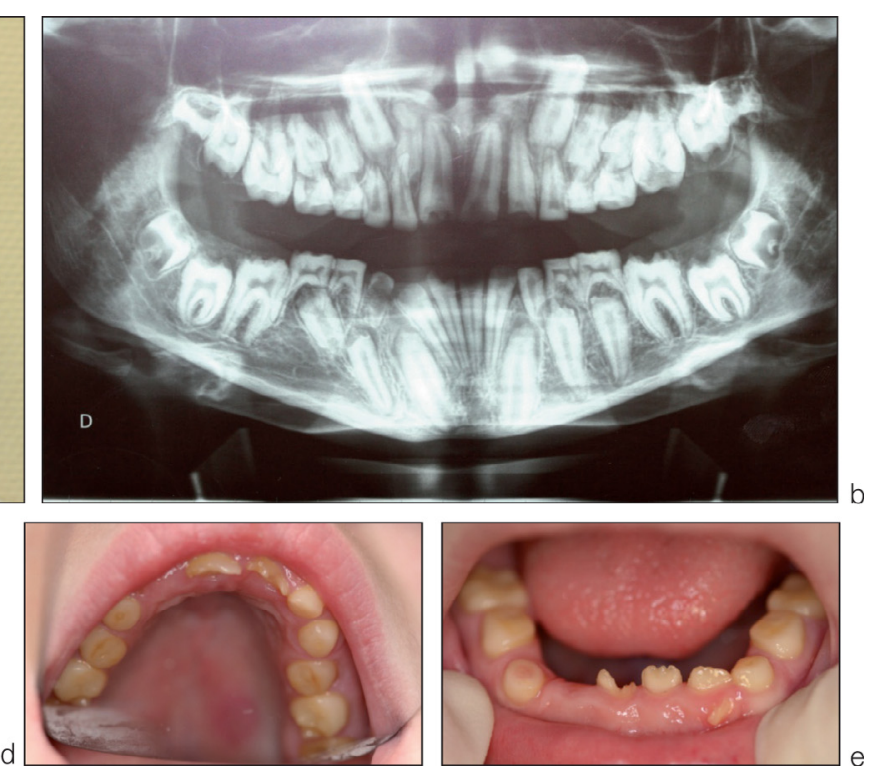

Figure 12

9 year old patient presenting with junctional epidermolysis bullosa:

a: photograph of the face;

b: panoramic radiograph highlighting anomalies of enamel of all the teeth as well as significant delay in eruption;

$c, d, \&$ e: intraoral photographs showing severe generalized enamel hypoplasia affecting all the teeth.

can be normal or severely reduced leading to significant wear during mastication. Patients generally con- sult for the unesthetic appearance of the teeth and children are frequently teased at school. 
Most of the time, this pathology is isolated. From a genetic point of view, there are 7 genes that are responsible for the isolated forms (amelogenin, enameline, kallikrein 4, enamelysine, WDR32, FAM83H, C4orf26). On the other hand, other dental and extradental anomalies can be added.

Other syndromes can be associated with amelogenesis imperfecta. These syndromes more severely affect other organs, the diagnosis is made early by pediatricians and other specialists at birth and during infancy:

- Kohlschütter-Tönz syndrome is characterized by epilepsy and mental degeneration;

- Junctional epidermolyis bullosa is characterized by excessive

\section{4 - ANOMALIES OF THE DENTIN}

Hereditary osteogenesis imperfecta

(Fig. 13)

\section{Oro-facial clinical signs:}

- opalescent brownish gray teeth;

- short crown height;

- enamel fractures;

- multiple wear facets;

- short and thin roots;

- partial or total obliteration of the pulp chambers;

- periapical lesions;

- Class III malocclusion.

\section{Extra-oral signs:}

- blue sclera;

- loose ligaments; cutaneous and mucosal fragility generating the formation of bullae which rub off the dermis/ epidermis at the slightest friction;

- Tricho-dental-osseous syndrome is characterized by osseous anomalies (increase in the osseous density especially in the cranial bones), hair anomalies (thin, curly at birth and stiffening with age) and taurodontism of molars;

- Jalilli syndrome is characterized by a dystrophy of the cones and rods of the eyes leading to a loss of central vision, loss of color vision and visual acuity with photophobia that can lead to blindness.

- bone fractures;

- deformation of the long bones;

- deafness;

- small size;

- thickening of the cranial perimeter with occipital protruberance;

- radiological appearance or wormien wormian bones.

This is a genetic disease that in the majority of cases qualitatively or quantitatively alters the osseous matrix following an alteration of the gene coding for type I collagen. This pathology results from an osseous fragility that is associated with an increase in the number of fractures during childhood. In the most severe forms the bones present with deformations and significant deficits in 

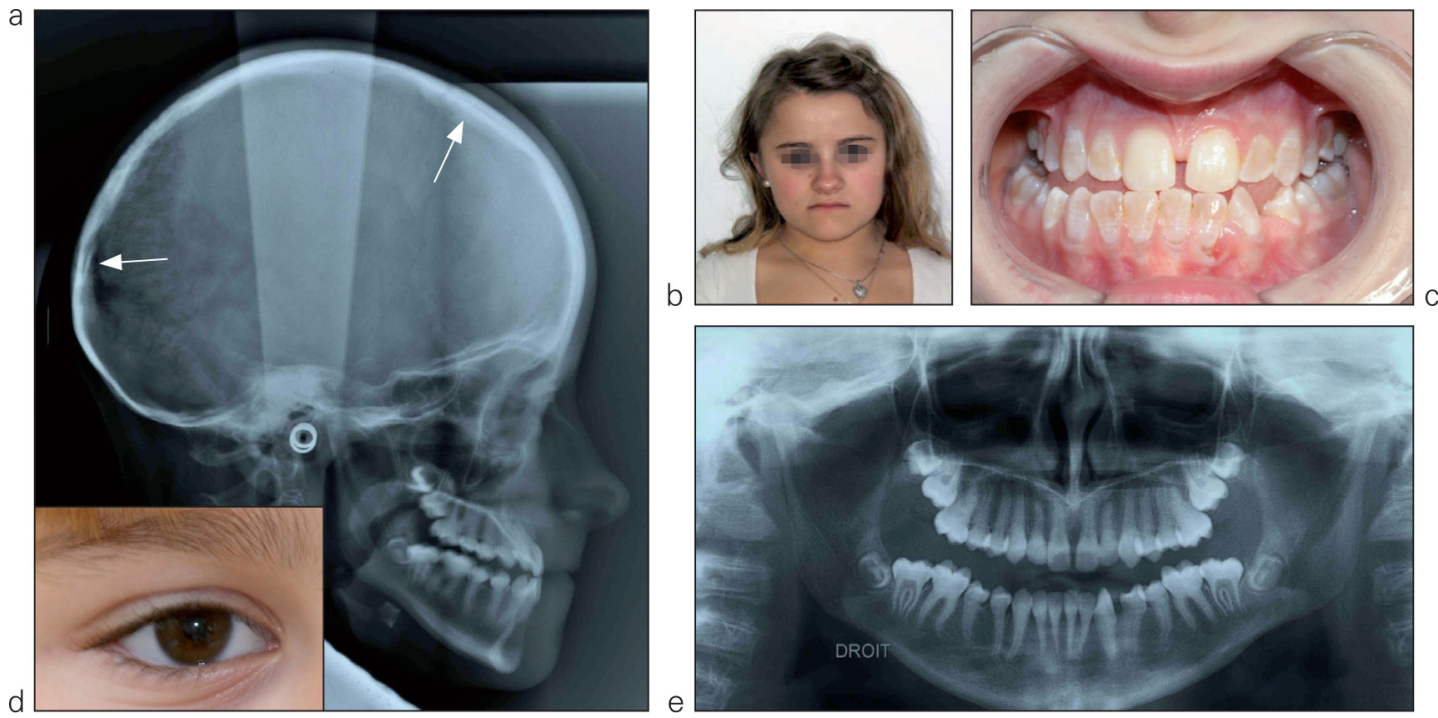

Figure 13

12 year old patient presenting with osteogenesis imperfecta associated with hereditary dentinogenesis imperfecta:

a: lateral profile xray showing a skeletal Class III, some wormian bones of the cranial vault;

b: facial photograph;

c: intraoral photograph showing the gray opalescent shade of the permanent teeth;

d: Eye photograph showing the blue sclera blue sclera;

e: panoramic radiograph showing the characteristic aspect of the teeth with dentinogenesis imperfecta (globular crowns, thin roots, obliterated pulp chambers).

growth are observed. Lethal forms are described. Type I osteogenesis imperfecta is the least severe form, and can go unnoticed. These patients simply present with an increased number of osseous fractures.

In the dental arena, these patients present with dentinogenesis imperfecta. This is a genetic disease altering the structure of dentin (type I collagen represents $90 \%$ of the protein content of the dentinal matrix). The primary and permanent teeth are both afflicted with, on the other hand, a more significant severity afflicting the primary teeth. On the cranio-facial plane area a more significant prevalence of Class III malocculsions is noted ${ }^{8}$. When facing such a clinical picture, it is therefore necessary to look for an osteogenesis imperfecta, by using osseous densitometry (that should be dose-decreased if the patient presents with osteogenesis imperfecta). If the osseous densiometry is normal, the positive diagnosis will then be isolated hereditary dentinogenesis imperfecta, a pathology in all ways similar but affecting only the teeth and due to another dental gene, the DSPP. 


\section{5 - HYPOCALCIFICATION}

\section{Oro-facial clinical signs:}

- premature loss of primary teeth;

- non-traumatic loss of permanent teeth.

\section{Extra-oral signs:}

- respiratory problems;

- premature craniosynostosis;

- extended osseous demineraliztions;

- rickets (delayed growth and bone deformity) of the metaphyses;

- skeletal malformations;

- short size and difficulty walking (waddling gait);
- bony fractures;

- chondrocalcinosis (adult);

- osteoarthropathy(adult).

Hypocalcification is a disease linked to a deficit in alkaline phosphatase protein, an enzyme that contributes to the mineralization of osseous and dental tissues. The most severe form (peri-natal) is lethal with the absence of skeletal mineralization. On the other hand, in the least severe form, only dental anomalies are present. The determination of a low level of activity of this enzyme in these patients is a diagnostic criterion.

\section{6 - ANOMALIES OF MAXILLO-FACIAL GROWTH}

\section{Wiedemann-Beckwith syndrome}

\section{Clinical oro-facial signs:}

- Macroglossia;

- exophthalmia associated with a infra-orbital fold;

- port-wine stain;

- underdevelopment of the middle third of the face;

\section{Extra-oral clinical signs:}

- anomalies of development of the visceral walls (omphalocele, umbilical hernias);

- fetal macrosomia (birth weight $>4 \mathrm{~kg}$ );

- gigantism;

- visceromegaly;

- cardiomegaly;

- hypoglycemia (can be lethal).

Wiedemann-Beckwith syndrome is a developmental anomaly character- ized by excessive growth and a predisposition to the development embryologic tumors. Its incidence is $1 / 13,700$ births and of its expression is extremely variable among patients ranging from unnoticeable features to forms that are lethal at birth. Close to $85 \%$ are sporadic but familial cases have also been reported. This excessive growth begins in the second half of the intra-uterine period and continues during the first years of life. In adults, on the other hand, these patients present a normal size. The majority of the tumors develop towards the age of 8-10 years as Wilms Tumors (nephroblastomas) or hepatoblastomas. From a genetic point of view, this pathology is heterogenous resulting from genetic or epigenetic alterations from genes that regulate growth, and carried on the $11 p 15.5$ chromosome ${ }^{11}$. 


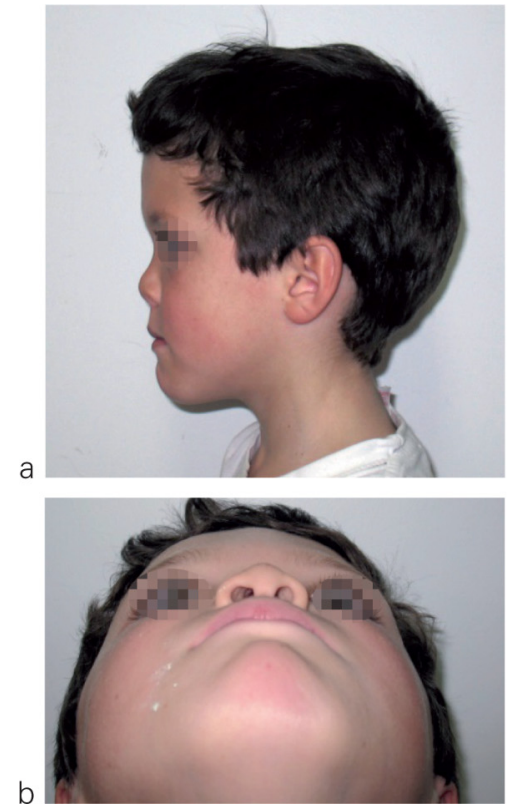

Patient presenting with Binder's syndrome:

$c$ : lateral profile $x$-ray showing underdeveloped jaws.

Binder's Syndrome (Fig. 14)

\section{Clinical oro-facial signs:}

- midfacial hypoplasia;

- underdeveloped jaws;

- short nose;

- Aplastic anterior nasal spine;

- Missing nasal edge;

- Nasal mucosal atrophy;

- Skeletal and dental Class III.

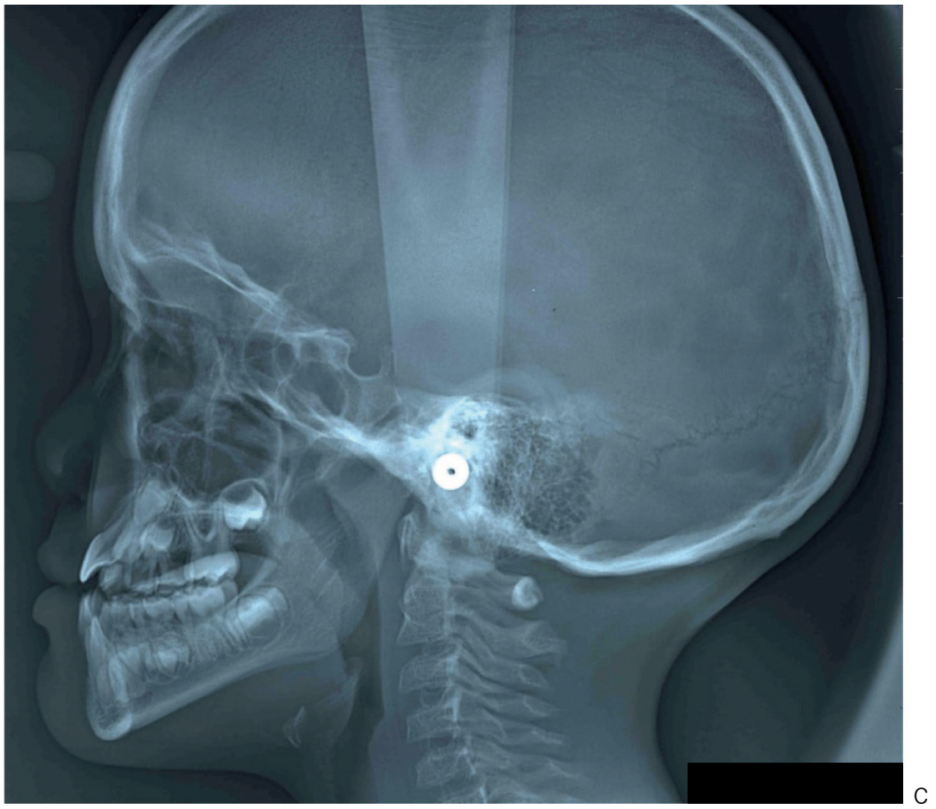

Figure 14

\section{Extra-oral signs:}

- Binder's syndrome, also called maxilla-nasal dysplasia, is essentially characterized by an anomaly of cranio-facial development located in the mid-face. The incidence estimated at $1 / 10,000$ is most likely underestimated. Its diagnosis is essentially made according to the maxillo-facial examination. Patients afflicted with this syndrome have a characteristic concave face. The nasal anomalies (due to the absence of nasal bone) are also a major diagnostic sign. The severity is extremely variable and the etiology is unknown ${ }^{9}$.

\section{Hemi-facial microsomia or oto-mandibular dysplasias}

Oto-mandibular dysplasia is characterized by an anomaly in of the development of the temporal and pterygo-mandibular complexes thus generating hypoplasias of the whole face maxilla, mandible and ears. It is most often unilateral and can be 
associated with other anomalies giving rise to multiple clinical forms and different syndromes.

Mandibular and articular malformations are major elements of the diagnosis. In the mandible a condylar hypoplasia can be responsible for multiple growth malformations (facial asymmetry, cant of occlusal plane). As for the ears, the anomalies vary from a simple pre-tragal tubercle to a hypoplasia of the middle ear. These malformations can be accompanied by anomalies of the surrounding tissues (hypoplasia of a portion of facial cranial nerves, atrophy of the soft tissue) and often deafness. Anomalies of the central nervous system or the cardiac system may also be observed but less frequently ${ }^{2}$.

Conflicts of interest: none

\section{REFERENCES}

1. Bennaceur S, Petavy-Blanc A, Chauve J, Louafi S, Hanss J. Morphologie céphalique humaine. Données anthropométriques du vivant. EMC Stomatologie 2005;22-001-D10, 85-103, Elsevier Masson.

2. Bettega G, Morand B, Lebeau J, Raphaël B. Morphological alterations of oto-mandibular syndromes. Annales de chirurgie plastique et esthétique 2001;46(5):495-506.

3. Bohring A, Stamm T, Spaich C, Haase C, Spree K, Hehr U, et al. WNT10A mutations are a frequent cause of a broad spectrum of ectodermal dysplasias with sex-biased manifestation pattern in heterozygotes. American Journal of Human Genetics 2009;85(1):97-105.

4. Chong AK. Common congenital hand conditions. Singapore Medical Journal 2010;51(12):965-71.

5. De Coster PJ, Marks LA, Martens LC, Huysseune A. Dental agenesis: genetic and clinical perspectives. Journal of Oral Pathology 2009;38(1):1-17.

6. Jaureguiberry G, De la Dure-Molla M, Parry D, Quentric M, Himmerkus N, Koike T, et al. Nephrocalcinosis (enamel renal syndrome) caused by autosomal recessive FAM20A mutations. Nephron Physiology 2012;122(1-2):1-6.

7. Jeanpierre M., Jonveaux P., Lacombe D., Leporrier N., Lyonnet S. MC. Approche de I'enfant dysmorphique. Génétique Médicale. Masson 2004;217-28.

8. Kamoun-Goldrat AS, Le Merrer MF. [Osteogenesis imperfecta and dentinogenesis imperfecta: diagnostic frontiers and importance in dentofacial orthopedics]. L' Orthodontie française. EDP Sciences; 2007;78(2):89-99.

9. Nedev PK. The Binder syndrome: review of the literature and case report. International Journal of Pediatric Otorhinolaryngology 2008;72(10):1573-6.

10. Roelfsema NM, Cobben JM. The EEC syndrome: a literature study. Clinical Dysmorphology 1996;5(2):115-27.

11. Weksberg R, Shuman C, Beckwith JB. Beckwith-Wiedemann syndrome. European Journal of Human Genetics: EJHG 2010;18(1):8-14.

12. Wright JT, Morris C, Clements SE, D'Souza R, Gaide O, Mikkola M, et al. Classifying ectodermal dysplasias: Incorporating the molecular basis and pathways (Workshop II). American Journal of Medical Genetics. Part A. 2009;149A(9):2062-7. 